\title{
Velocity fields in and around sunspots at the highest resolution
}

\section{Carsten Denker and Meetu Verma}

\author{
Astrophysikalisches Institut Potsdam, An der Sternwarte 16, D-14482 Potsdam, Germany
} email: cdenker@aip.de and mverma@aip.de

\begin{abstract}
The flows in and around sunspots are rich in detail. Starting with the Evershed flow along low-lying flow channels, which are cospatial with the horizontal penumbral magnetic fields, Evershed clouds may continue this motion at the periphery of the sunspot as moving magnetic features in the sunspot moat. Besides these well-ordered flows, peculiar motions are found in complex sunspots, where they contribute to the build-up or relaxation of magnetic shear. In principle, the three-dimensional structure of these velocity fields can be captured. The line-of-sight component of the velocity vector is accessible with spectroscopic measurements, whereas local correlation or feature tracking techniques provide the means to assess horizontal proper motions. The next generation of ground-based solar telescopes will provide spectropolarimetric data resolving solar fine structure with sizes below $50 \mathrm{~km}$. Thus, these new telescopes with advanced post-focus instruments act as a 'zoom lens' to study the intricate surface flows associated with sunspots. Accompanied by 'wide-angle' observations from space, we have now the opportunity to describe sunspots as a system. This review reports recent findings related to flows in and around sunpots and highlights the role of advanced instrumentation in the discovery process.
\end{abstract}

Keywords. Sun: atmospheric motions, Sun: photosphere, Sun: sunspots, Sun: magnetic fields

\section{Probing the velocity fields in and around sunspots}

New instruments and observing capabilities have advanced our knowledge about plasma motions in and around sunspots and their interaction with the magnetic fields. Therefore, by introducing current and future instruments for high-resolution studies of the Sun, we will set the stage for this review of the intricate flow fields of sunspots. The line-of-sight velocity can be derived from a Doppler-shifted spectral line profile, and its height dependence can be inferred by carefully selecting lines, which originate at different layers in the solar atmosphere, or by measuring the bisectors of spectral lines. Local correlation or feature tracking are the methods of choice in determining horizontal proper motions (see Fig. 1). In principle, spectroscopic and imaging techniques provide access to the three-dimensional velocity field.

Instruments commonly used to measure solar velocity fields can be placed into five broad categories ordered according to their spectral resolving power: (1) imaging with interference filters, (2) imaging with Lyot filters, (3) line-of-sight velocity and magnetic field measurements using filtergraphs, (4) imaging spectropolarimeters, and (5) long-slit spectrographs. We introduce observations obtained with the instruments ${ }^{1,2,3,5}$ (superscripts indicate the instrument category) of the Hinode Solar Optical Telescope (SOT, Tsuneta et al. 2008), the Interferometric Bidimensional Imaging Spectrometer (IBIS ${ }^{4}$, Cavallini 2006), the Crisp Imaging Spectropolarimeter (CRISP ${ }^{4}$, Scharmer 2006), the Göttingen/GREGOR Fabry-Pérot Interferometer (GFPI ${ }^{4}$, Bello González \& Kneer 2008; 


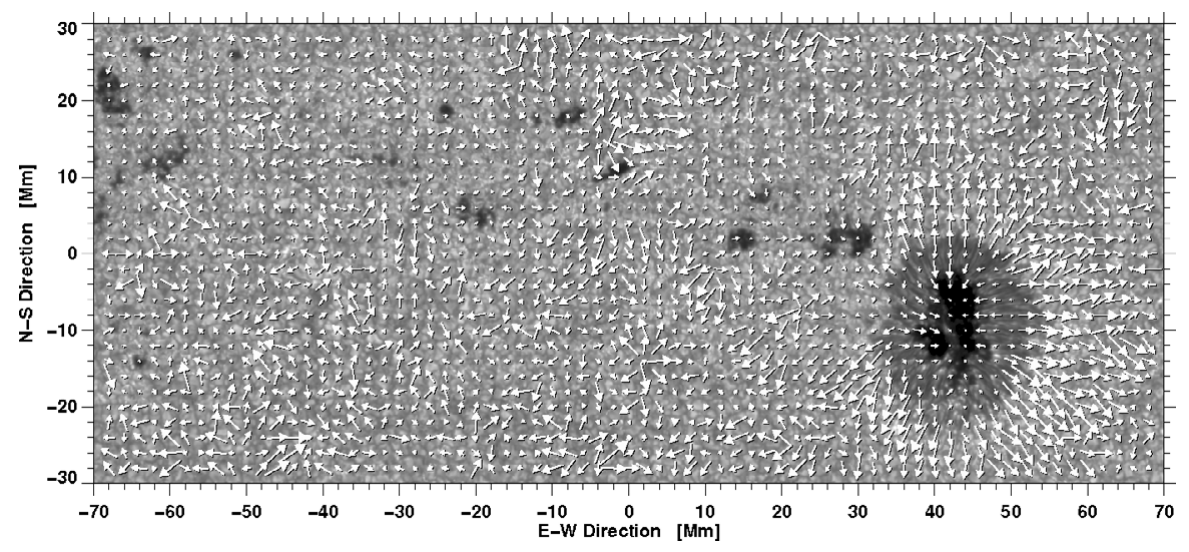

Figure 1. The Japanese Hinode mission placed for the first time a telescope into space, which can resolve the fine structure of sunspots. SOT feeds an instrument suite for high-resolution imaging and spectropolarimetry. These novel instruments provide access to the flow fields in and around sunspots. Displayed is a G-band image of active region NOAA 10921 observed near disk center on 2006 November 3. Direction and magnitude of horizontal flows are indicated by vectors. These high-resolution data set the stage for this review providing motivation for the next generation of ground-based telescopes and instruments.

Denker et al. 2010), the Tenerife Infrared Polarimeter (TIP ${ }^{5}$, Collados et al. 2007), and the Polarimetric Littrow Spectrograph (POLIS ${ }^{5}$, Beck et al. 2005).

The recent success of the Japanese Hinode mission (Kosugi et al. 2007) has certainly proven that high resolution observations pave the way for advancing solar physics. On the ground first observations have been obtained with the New Solar Telescope (NST, Denker et al. 2006; Goode et al. 2010), while the GREGOR solar telescope (Volkmer et al. 2010) awaits commissioning upon delivery of its primary mirror. With the Advanced Technology Solar Telescope (ATST, Rimmele et al. 2010) beginning construction and the European Solar Telescope (EST, Collados et al. 2010) finishing the design and development phase, further progress and new discoveries are expected when approaching the fundamental spatial scales for physical processes on the Sun.

A short review can never be complete but we at least strove to be up-to-date. Consequently, our approach had to be a very subjective one motivated by the imminent commissioning of the GREGOR solar telescope and the upcoming science demonstration time with the GFPI.

\section{The intricate flow fields of simple sunspots}

\subsection{Convective signatures in the umbra}

The existence of convective signatures in the umbra has been debated for many years. High-resolution observations with CRISP pinpointed strong upflows of up to $1.4 \mathrm{~km} \mathrm{~s}^{-1}$ in deep photospheric layers, which are associated with bright umbral dots (Ortiz et al. 2010). The height dependence of line-of-sight velocities was determined from the bisectors of coarsely sampled spectral lines. The umbral dots are identified with columns of hot, rising material with weaker and more inclined magnetic fields as compared to the umbral background. To establish overturning convective energy transport in the umbra, downflows have to exist, which were indeed observed $\left(0.4-1.0 \mathrm{~km} \mathrm{~s}^{-1}\right)$ in confined areas at the periphery of umbral dots as predicted by theory. Some elongated umbral dots 
exhibit dark lanes in their centers, where the magnetic field is even weaker and more inclined. These umbral dots resemble 'coffee beans' because of this peculiar intensity pattern, which originates from the accumulation of material at the top of rising plumes increasing the density and elevating the opacity (Schüssler \& Vögler 2006). As pointed out by Ortiz et al. (2010), the velocity gradient with height in umbral dots imposes an observational challenge, i.e., the Doppler shifts are hard to observe in the line core - explaining the scarcity of reported umbral downflows, which are a phenomenon of the deep photosphere. In addition, small-scale features such as umbral dots change size and velocity within a few minutes, thus necessitating observations with high spatial and temporal resolution.

Scrutinizing light-bridges separating different umbral cores, Rouppe van der Voort et al. (2010) found another remarkable feature. Even though similar in appearance to granulation, light-bridges have dark central lanes with a width of about $400 \mathrm{~km}$, where upflows reside, which are strongest in deep photospheric layers. Typical velocities are around $0.5 \mathrm{~km} \mathrm{~s}^{-1}$ but reach as high as $1.0 \mathrm{~km} \mathrm{~s}^{-1}$. However, the direction of the flows is just opposite to the downflows in intergranular lanes. Similar to the dark lanes in umbral dots, the narrow, dark channels of light-bridges have weaker and more inclined magnetic fields.

\subsection{Penumbral fine structure and Evershed flow}

In the penumbra, the Evershed flow denotes a radial outflow of gas, which is aligned with more horizontally placed magnetic flux tubes in dark penumbral filaments. Theoretical models based on the thin flux tube approximation arrive at steady as well as time-dependent flows driven by pressure differences. The 'moving flux tube' model (e.g, Schlichenmaier 2002) explains much of the observed fine structure. However, 'steady siphon-flow' models still remain viable and provide strong arguments against superAlfvénic, 'sea-serpent'-like flows (Thomas 2005), namely that they are gravitationally unstable.

The average vertical velocity field of the quiet Sun as observed by the Hinode spectropolarimeter (Franz \& Schlichenmaier 2009) is always dominated by upflows, whereas the penumbra shows a different behavior. Here, upflows cover a larger area for Doppler velocities below $0.4 \mathrm{~km} \mathrm{~s}^{-1}$, in particular in the inner penumbra. However, at velocities above $0.6 \mathrm{~km} \mathrm{~s}^{-1}$ downflows have a larger areal coverage. This leads to a net downflow of more than $0.1 \mathrm{~km} \mathrm{~s}^{-1}$ for the entire penumbra. The upflows of the inner penumbra are typically elongated an possess an aspect ratio of about five. In contrast, the largest downflows in the penumbra of up to $9 \mathrm{~km} \mathrm{~s}^{-1}$ at the outer penumbral boundary exceed even the largest quiet Sun values of about $3 \mathrm{~km} \mathrm{~s}^{-1}$ and have a shape closer to circular.

Penumbral grains migrate inwards in the inner penumbra. The Evershed flow begins at the leading edge of the penumbral grains (Ichimoto et al. 2007), which have been identified with the footpoints of hot upflows in strongly inclined flux tubes (Rimmele \& Marino 2006). The Evershed flow then turns horizontal and follows preferentially the dark cores of the penumbral filaments (Scharmer et al. 2002), where the magnetic field is more horizontal. The striking dark-cored penumbral filaments are much easier to discern in polarized light than in continuum images (Bellot Rubio et al. 2007). Their spectral line profiles are very asymmetric, which hints at multiple magnetic field components along the line-of-sight or within the resolution element. The dark cores have a lateral extend of less than $200 \mathrm{~km}$ and exhibit magnetic fields, which are weaker by 100-150 G as compared to the lateral brightenings. The Evershed flow reaches velocities up to and even exceeding the photospheric sound speed of about $7 \mathrm{~km} \mathrm{~s}^{-1}$. The Evershed flow shows 
temporal variations on scales of 10-15 min, which also corresponds to the intensity variations of Evershed clouds. Small patches of opposite polarity and strong downflows are observed throughout the outer penumbra indicating that some penumbral field lines already return to the interior well within the penumbra itself (Sainz Dalda \& Bellot Rubio 2008). Selecting spectral lines with contribution functions covering the deep photosphere or bisector analysis are the means to determine the height dependence of the Evershed flow, which increases in strength with depth. The Evershed flow is not stationary. Coherent flow patches (Evershed clouds) can be traced from within the sunspot to move away from the spot (Cabrera Solana et al. 2006), where they can be associated with moving magnetic features (MMFs).

\subsection{Moat flow and moving magnetic features}

Martínez Pillet et al. (2009) report that the Evershed flow continues at least sporadically outside the penumbra into the sunspot moat, i.e., not all field lines, which carry the Evershed flow, submerge below the photosphere at the penumbra's outer boundary. Analyzing spectropolarimetric Hinode data, Shimizu et al. (2008) find high-speed (supersonic) downflows by analyzing Stokes- $V$ profiles. If such profiles become more complicated, i.e., if they have multiple lobes, then using the zero-crossing of the Stokes- $V$ profile is not a good indicator of flow velocities. This argues in favor of instruments capable of resolving spectral line profiles and not filtergraph systems, which deliver Dopplergrams and magnetograms prone to erroneous interpretation. The observed downflows occur in three distinct locations: (1) the outer penumbral boundary (MMFs), (2) the edge of the umbra in absence of penumbral structures, and (3) near small-scale field concentrations in the sunspot moat (convective collapse). These features have in common a pointlike appearance with diameters of about $1^{\prime \prime}$. They are transient features with lifetimes from a few minutes to about $30 \mathrm{~min}$ but on average the lifetimes tend towards the lower end of this range. On the other hand, in long-duration observations, filamentary magnetic features become visible in the sunspot moat (Sainz Dalda \& Martínez Pillet 2005) revealing that MMFs preferentially move along certain pathways. Balthasar and Muglach (2010) present another interesting finding, namely that in the inner moat the flow velocities are higher in the ultra-violet $(170 \mathrm{~nm})$ as compared to visible $(500 \mathrm{~nm})$ continuum, while reversing this relationship in the outer moat.

Active region NOAA 10977 contained a bipolar group of pores, which never developed a penumbra, even though infrared Ca II $\lambda 854.2 \mathrm{~nm}$ observations revealed superpenumbral structures (a worthwhile research topic on its own). In a coordinated observing campaign with Hinode and IBIS, Zuccarello et al. (2009) detected short, radially aligned magnetic structures at the periphery of the pore, which however did not bear any resemblance to penumbral filaments. Consequently, in their absence no indications of the Evershed effect could be detected. Surprisingly, both moat flow and MMFs were surrounding the pore questioning their close ties to the Evershed flow. The presence of MMFs was interpreted as twisted magnetic field lines, which were pealed away from the vertical flux bundle of the pore by the (super)granular flow.

\subsection{Decay of sunspots}

A mechanism to remove magnetic flux from a sunspot is the interaction of penumbral filaments at the edge of the sunspot with the granulation, which erodes the magnetic field of the sunspot (Kubo et al. 2008). Flux detaches in the form of MMFs from the more vertical background fields of the uncombed penumbra, thus contributing to the decay of sunspots. Other types of MMFs, which are related to the magnetized Evershed flow, do not change the net magnetic flux. These dark penumbral filaments with strong horizontal 
magnetic fields often reach into the moat region. This goes along with a non-stationary penumbral boundary, which advances and retracts with respect to an average position.

In the last stage of sunspot decay, only a pore without a penumbra remains (Bellot Rubio et al. 2008), where small finger-like, weak, and almost horizontal magnetic features of opposite polarity can be recognized at the pore's boundary. They extend up to about 1.5 Mm and have blue-shifted Stokes profiles indicative of upflows with speeds of 1$2 \mathrm{~km} \mathrm{~s}^{-1}$. This could be the remnants of magnetic field lines, which previously carried the Evershed flow. No longer held down by the mass provided by the Evershed flow, they become boyant and lift of to vanish in the chromosphere. In general, the question remains open, where penumbral flux tubes end. At least in the final stages of a decaying sunspot, alternatives might exist to the notion that they just bend below the surface at the periphery of the penumbra. This problem is also tied to the matter of mass continuity in the Evershed flow, since sources and sinks still need to be unambiguously identified and the balance between inflows and outflows has to be established. If the mass would be supplied by the flux rope rising through the convection zone, which initially led to the emergence of the sunspot, then decoupling from this flux rope would shut off the mass flow causing the sunspot to decay. It is noteworthy that the divergence line observed in the middle penumbra survives the decay process (Deng et al. 2007) and even the moat flow is still detectable long after the penumbra has vanished (Zuccarello et al. 2009).

\section{Peculiar flows in the context of eruptive events}

Flares occur near magnetic neutral lines, where strong magnetic field gradients exist, and where the horizontal component of the magnetic field is strongly sheared. The height dependence of horizontal proper motions can be derived by applying local correlation tracking techniques to images obtained in multiple spectral regions. Near-infrared images at the opacity minimum probe the deepest photospheric layers, whereas G-band images provide access to higher layers as compared to continuum images observed in the visible part of the solar spectrum. Deng et al. (2006) presented such a multi-wavelength study of active region NOAA 10486 - one of the most flare-prolific regions of solar cycle 23 . Both horizontal and vertical shear flows with speeds of about $1 \mathrm{~km} \mathrm{~s}^{-1}$ exist in the vicinity of magnetic neutral lines. These flows are long-lived and persistent. Thus, the magnetic field might not be the only agent trigerring solar flares. The flow speed in shear regions is diminishing with height while the direction essentially the same. Therefore, shear flows are dominant in the deeper layers of the photosphere. In response to an X10 flare the shear flows significantly increased, which was interpreted as shear release in the overlying magnetic fields or as the emergence of twisted and sheared flux infusing energy from subphotospheric layers. Shear flows are not just limited to horizontal flow fields. In a spectropolarimetric study of a B7.8 flare in active region NOAA 10904, Hirzberger et al. (2009) detected (supersonic) downflows of up to $7 \mathrm{~km} \mathrm{~s}^{-1}$ in the penumbra. Twisting and interlaced penumbral filaments are no longer radially aligned with respect to the major sunspot and become almost tangential. However, the Evershed flow remains aligned with the magnetic field. Islands of opposite polarity and complex flows require a threedimensional topology necessitating high spectral resolution to capture these features in multi-lobed Stokes- $V$ profiles.

Twisted and sheared penumbral filaments, photospheric shuffling of footpoints, and rapid motion as well as rotation of sunspots can all destabilize the magnetic fields above an active region. In a multi-wavelength study of active region NOAA 10960, Kumar et al. (2010) find evidence that helical twist is accumulated before the flare and then activated to release the stored energy during the flare. The orientation of penumbral filaments 
can strongly deviate from the radial direction in satellite sunspots and $\delta$-configurations. Photospheric signatures of the flare are the now well-established rapid penumbral decay and umbral enhancement (Liu et al. 2005), which are indicative of a rearranged magnetic field topology. In addition to these signatures, Gosain et al. (2009) noticed the lateral displacements of penumbral filaments during an X3.4 flare in active region NOAA 10930 on 2006 December 13. This occurs immediately before (4 min) the flare initiation, when penumbral filaments move laterally towards the magnetic neutral line, while changing direction and moving away from it for about $40 \mathrm{~min}$ after the flare. The energy involved in the lateral displacements is only a few percent of the total energy released in the flare.

\section{Conclusions}

Space observations are not only free from seeing but, depending on orbit, do not have to cope with the day night-cycle on Earth. Thus, in principle, high cadence observations with long coverage become possible to follow magnetic structures of active regions while they evolve. Such observations from space are only limited by on-board processing and telemetry. Despite the availability of long time-series with consitstent quality only few systematic and comprehensive studies of flow fields are available. The majority of investigations have been limited to case studies. This is an opportunity for the time yet to come to explore this enormous database with the aim to put the still fragmented puzzle of flows in and around sunspots together into a comprehensive picture. In this respect, high-cadence vector magnetograms of the recently launched Solar Dynamics Observatory (SDO) will certainly advance our knowledge regarding eruptive events on the Sun.

The new and upcoming instrumental capabilities on the ground will drastically improve angular resolution. Adaptive optics and future multi-conjugate adaptive optics will allow to capture time-series of spectropolarimetric data, which are longer than the lifetime of solar (small-scale) structures. The polarimetric sensitivity and spectral resolution of these instruments including infrared capabilities will produce multi-dimensional data sets suitable for advanced spectral inversion techniques. For the first time, physical quantities become accessible at the fundamental scales of physical processes on the Sun.

\section{Acknowledgements}

MV expresses her gratitude for the generous financial support by the German Academic Exchange Service (DAAD) in the form of a PhD scholarship. CD acknowledges a DAAD travel grant facilitating his attendance at the IAU Symposium.

\section{References}

Balthasar, H. \& Muglach, K. 2010, Astron. Astrophys 511, A67

Beck, C., Schmidt, W., Kentischer, T., \& Elmore, D. 2005, Astron. Astrophys 437, 1159

Bello González, N. \& Kneer, F. 2008, Astron. Astrophys 480, 265

Bellot Rubio, L. R., Tritschler, A., \& Martínez Pillet, V. 2008, Astrophys. J. 676, 698

Bellot Rubio, L. R., Tsuneta, S., Ichimoto, K., et al. 2007, Astrophys. J. Lett. 668, L91

Cabrera Solana, D., Bellot Rubio, L.R., Beck, C., \& del Toro Iniesta, J.C. 2006, Astrophys. J. $649, \mathrm{~L} 41$

Cavallini, F. 2006, Solar Phys. 236, 415

Collados, M., Lagg, A., Díaz García, J.J., et al. 2007, ASP Conf. Ser. 368, 611

Collados, M., Bettonvil, F., Cavaller, L., et al. 2010, Proc. SPIE 7733, 77330H

Deng, N., Xu, Y., Yang, G., et al. 2006, Astrophys. J. 644, 1278

Deng, N., Choudhary, D.P., Tritschler, A., et al. 2007, Astrophys. J. 671, 1013.

Denker, C., Goode, P.R., Ren, D., et al. 2006, Proc. SPIE 6267, 62670A 
Denker, C., Balthasar, H., Hofmann, A., et al. 2010, Proc. SPIE 7735, 77356M

Franz, M. \& Schlichenmaier, R. 2009, Astron. Astrophys 508, 1453

Goode, P.R., Yurchyshyn, V., Cao, W., et al. 2010, Astrophys. J. Lett. 714, L31

Gosain, S., Venkatakrishnan, P., \& Tiwari, S.K. 2009, Astrophys. J. Lett. 706, L240

Hirzberger, J., Riethmüller, T., Lagg, A., et al. 2009, Astron. Astrophys 505, 771

Ichimoto, K., Suematsu, Y., Tsuneta, S., et al. 2007, Pub. Astron. Soc. Jap. 318, 1597

Kosugi, T., Matsuzaki, K., Sakao, T., et al. 2007, Solar Phys. 243, 3

Kubo, M., Lites, B.W., Ichimoto, K., et al. 2008, Astrophys. J. 681, 1677

Kumar, P., Srivastava, A.K., Filippov, B., \& Uddin, W. 2010, Solar Phys. 266, 39

Liu, C., Deng, N., Liu, Y., et al. 2005, Astrophys. J. 622, 722

Martínez Pillet, V., Katsukawa, Y., Puschmann, K.G., \& Ruiz Cobo, B. 2009, Astrophys. J. Lett. 701, L79

Ortiz, A., Bellot Rubio, L.R., \& Rouppe van der Voort, L. 2010, Astrophys. J. 713, 1282

Rimmele, T.R. \& Marino, J. 2006, Astrophys. J. 646, 593

Rimmele, T.R., Wagner, J., Keil, S., et al. 2010, Proc. SPIE 7733, 77330G

Rouppe van der Voort, L., Bellot Rubio, L.R., \& Ortiz, A. 2010, Astrophys. J. Lett. 718, L78

Sainz Dalda, A. \& Bellot Rubio, L.R. 2008, Astron. Astrophys 481, L21

Sainz Dalda, A. \& Martínez Pillet, V. 2005, Astrophys. J. 632, 1176

Scharmer, G.B. 2006, Astron. Astrophys 447, 1111

Scharmer, G.B., Gudiksen, B.V., Kiselman, D., et al. 2002, Nature 420, 151

Schlichenmaier, R. 2002, Astron. Nachr. 323, 303

Schüssler, M. \& Vögler, A. 2006, Astrophys. J. Lett. 641, L73

Shimizu, T., Lites, B.W., Katsukawa, Y., et al. 2008, Astrophys. J. 680, 1467

Thomas, J.H. 2005, Astron. Astrophys 440, 29

Tsuneta, S., Ichimoto, K., Katsukawa, Y., et al. 2008, Solar Phys. 249, 167

Volkmer, R., von der Lühe, O., Denker, C., et al. 2010, Proc. SPIE 7733, 77330K

Zuccarello, F., Romano, P., Guglielmino, S.L., et al. 2009, Astron. Astrophys 500, L5

\section{Discussion}

AULANIER: Very nice observations. It's very interesting. I have a question which is maybe delicate. You talked at the end of your presentation about space weather forecasting. So what do you think these nice high resolution and small focused on one sunspot can teach us about space weather?

DENKER: Okay. What we can do is try to understand what's happening within the regions - say, along the neutral line what is really happening there. I mean, if you look at space weather forecasting, people look at the neutral line do some crazy averages over sheer flows, magnetic sheer angle, and so on.

But if you look at the quantities, they don't tell you much about whether there will be a flare or not. I think the reason for that is because you don't understand what happens, let's say, more at the microscopic level. If you can bring instruments like the GFPI to any of space weather forecasting system, I doubt it as well.

But it will tell you, if there are certain regions and active regions which are more important than others - and you might actually need different instruments to do that you might not need the highest resolution once you know what feature you have to look at.

PRIEST: One question is to ask - what the main capabilities of GREGOR would be. And the second question was, when you're identifying the upflows and downflows in the penumbra, - can you roughly estimate there's mass balance between the two? In other words, whether you're seeing all of the upflows going down or whether some of them will continue into the moat. 
DENKER: Let's answer the last one first. I think it's difficult because many of the you probably won't see or haven't seen yet because, if you only see at the top, you might upflows because they are maybe just scale deeper. It's not much. It's 40 kilometers deeper. Then how can you answer that question? But there are lines where you can go deeper. For example, the infrared at 1.56 microns, and that maybe ties in a little bit with the first part of your question. The nice thing about GREGOR, you have a big aperture. Can actually get three times higher resolution than with Hinode - not all the time, I mean, you have seen. But maybe for two hours three hours a day. Will get actually data sets where it can follow small scale features. The aperture is one and a half meters. Three times larger than the Hinode. Also bigger than what we see with the Swedish telescope, and already there we see that fine structure has substructure. Hope is you actually get to the substructure as well, but the other thing I wanted to mention have the GFPI for visible light observations - we have the instrument or which is the new name for it, where you have infrared observation and you can do it at the same time. Is another thing you want to get as much information as possible, and our problem is you have just very small field of view. Let's just say 60 by 60 arc seconds. Need a space based instrument to get the big picture.

I think that's where all the synergies will be in the future to basically have something like a magnifying glass looking at the small details and then a space data to get the big picture and bring it all together. 